\title{
Valeur et prix des humanités
}

Professionnalisation et culture commune

Humanities Value and Awards: Professionalization and a Common Culture

\section{Yves Citton}

\section{OpenEdition}

\section{Journals}

Édition électronique

URL : http://journals.openedition.org/recherchestravaux/1548

DOI : $10.4000 /$ recherchestravaux.1548

ISSN : 1969-6434

Éditeur

UGA Éditions/Université Grenoble Alpes

Édition imprimée

ISBN : 978-2-37747-098-3

ISSN : 0151-1874

Référence électronique

Yves Citton, «Valeur et prix des humanités », Recherches \& Travaux [En ligne], 94 | 2019, mis en ligne le 20 juin 2019, consulté le 08 septembre 2020. URL : http://journals.openedition.org/ recherchestravaux/1548; DOI : https://doi.org/10.4000/recherchestravaux.1548

Ce document a été généré automatiquement le 8 septembre 2020

(c) Recherches \& Travaux 


\title{
Valeur et prix des humanités
}

\author{
Professionnalisation et culture commune
}

Humanities Value and Awards: Professionalization and a Common Culture

Yves Citton

1 Il est certainement urgent de s'interroger sur la valeur des enseignements en langues et en sciences humaines. Le sentiment commun est désormais que l'avenir de ces enseignements ne va nullement de soi : autant que la question de leur périmètre, qui a toujours été âprement débattue, c'est celle de leur survie même qui se pose au moins depuis quelques décennies, et de façon accélérée avec les transformations numériques. Aura-t-on encore besoin d'apprendre des langues étrangères lorsqu'il suffira de passer un PDF ou un e-book dans Google Translate pour en avoir aussitôt une (bonne) traduction dans sa langue propre, ou lorsque Siri affichera une traduction simultanée de ce que nous dit un passant interrogé dans la rue ? Aura-t-on du temps à perdre à enseigner la littérature lorsque les pressions de la compétitivité néolibérale auront intégralement " professionnalisé » nos cursus d'études?

2 Même si nos disciplines n'en finissent pas de tomber des nues, voilà déjà assez longtemps qu'on se demande pour combien de temps encore il sera considéré comme « rentable » de financer des enseignements en lettres, arts et sciences humaines - disciplines que je rangerai sous la catégorie générale d'« humanités » (au sens anglais de Humanities). Le problème plus pertinent me semble être aujourd'hui de comprendre quels appareils de mesure sont mobilisés pour répondre à une telle question, et à quelle échelle. Les "projets d'avenir» de nos disciplines doivent impérativement faire face à ces problèmes s'ils ne veulent pas bâtir l'avenir en question sur un château de sable. Je m'efforcerai ici de formuler ce problème à partir d'un exemple concret importé des États-Unis mais éclairant pour le cas français, malgré les différences évidentes et profondes qui séparent encore les deux côtés de l'Atlantique. 


\section{Petite remarque et grande enquête}

3 Le 30 janvier 2014, lors d'une conférence dans une usine de General Electric à Waukesha, dans le Wisconsin, le président Obama a dit que "les gens peuvent potentiellement gagner beaucoup plus avec de bonnes compétences manuelles et un apprentissage qu'ils ne pourraient le faire avec un diplôme en histoire de l'art ». Bien entendu, dès le lendemain, tout ce que les États-Unis comptent d'enseignants d'histoire de l'art, et plus généralement de praticiens et d'apologistes des Humanities, lui sont tombés sur le dos pour l'accuser de saper les valeurs fondamentales de l'éducation humaniste. Sitôt après avoir énoncé la phrase en question, visiblement improvisée, et anticipant immédiatement la réaction qu'elle allait déclencher, le président avait pourtant bien pris soin de préciser : «Bien sûr, il n'y a rien de mal à faire de l'histoire de l'art - j'adore l'histoire de l'art, et je ne veux pas recevoir des piles de courriels de toutes parts à ce sujet. Tout ce que je dis, c'est qu'on peut bien gagner sa vie et faire une belle carrière sans avoir de diplôme universitaire, pour autant qu'on acquière les compétences et l'apprentissage dont on a besoin ». Deux semaines plus tard, il devait encore prendre la peine de s'excuser par écrit envers un enseignant du Texas qui lui reprochait sa phrase malheureuse : «Je vous prie d'excuser ma remarque impromptue. Je parlais du marché de l'emploi, non de la valeur de l'histoire de l'art. Il se trouve que l'histoire de l'art était un de mes sujets favoris au lycée, et qu'elle m'a aidé à avoir des expériences d'épanouissement durant ma vie, à côté desquelles je serais passé sans elle1."

4 Un observateur distant (cruel? réaliste?) dirait que toute la différence entre les présidents G.W.Bush et Barack Obama tient à ce que le second a l'intelligence d'anticiper immédiatement les réactions causées par une telle phrase, puis la politesse de s'en excuser. Mais malgré leur rapport très différent à la culture, les deux présidents paraissent bien s'accorder sur un point - qui relève de leur époque plus que de leur idéologie : un parcours d'études constitue un investissement. On peut en tirer plus ou moins de revenus - même si ces revenus peuvent être de natures différentes (make more money, make a better living, take a greater deal of joy). Cela semble relever de l'évidence, et il faut toute l'hyper-sensibilité d'enseignants à la fois névrosés et paranoïaques pour y trouver à redire. Les familles, les bourses et/ou les prêts étudiants ne doivent-ils pas investir des milliers d'euros ou de dollars pour financer les années passées sur les bancs d'universités ? L'État ne compte-t-il pas ces investissements en termes de milliards? N'est-il pas raisonnable de mesurer et de comparer ce que les futurs salariés, ainsi que l'économie nationale, retireront de ces investissements?

5 Quoi qu'on pense des sentiments réels du président Obama, un deuxième phénomène culturel me parait à la fois aider à contextualiser sa petite phrase plus ou moins malheureuse, et fournir un cadrage beaucoup plus significatif quant aux «Projets d'avenir " des sciences humaines. Comme ils le font désormais chaque année, les analystes de l'entreprise PayScale viennent de publier la version 2014 de leur College ROI Report, consultable en ligne ${ }^{2}$, qui compare systématiquement les frais d'inscription payés pour accéder à une université nord-américaine (frais qui peuvent parfois se monter à 60000 dollars par an) et les salaires gagnés ultérieurement par les détenteurs des diplômes obtenus dans chaque institution. Nous sommes ici au cœur de la question posée plus haut puisque ROI sont les initiales de Return On Investment (retour sur investissement). 
Plutôt que d'en analyser les résultats, demandons-nous surtout comment fonctionne cette étude. Sans entrer dans les détails de collection des données, qui mériteraient une longue analyse, mentionnons seulement qu'il s'agit de Bachelor's degrees (BA), soit du diplôme qu'on obtient après environ quatre ans d'études undergraduate, ce qui ne correspond pas exactement à une licence française, si ce n'est que le BA permet comme la licence de s'inscrire pour des études graduate de master (MA) puis de doctorat (PhD). Une particularité de cette enquête est toutefois de ne tenir compte que des étudiants engagés dans un emploi à plein temps qui n'ont pas continué au-delà du BA - ce qui entraîne des distorsions considérables sur les résultats, distorsions généralement ignorées par les utilisateurs de ces rapports.

7 PayScale propose en effet sur son site Internet un formulaire permettant aux (parents des) futurs étudiants de spécifier un certain nombre de variables (l'État des États-Unis où l'on souhaite faire ses études, le type de parcours de spécialisation qu'on a en tête, le fait qu'on bénéficie ou non d'une bourse d'étude, qu'on souhaite habiter sur le campus ou non, etc.) afin de calculer quelles sont les institutions d'enseignement les plus rentables répondant à ces critères. L'échelle par défaut est déterminée par les retours sur investissements qu'apportera le diplôme visé, en termes de plus-value salariale sur les 20 ou 30 ans à venir - à partir d'anticipations basées sur les déclarations de salaire qui alimentent constamment la base de données rassemblée par PayScale. Sans diplôme de BA, les moyennes statistiques estiment que vous allez gagner une somme S; obtenir ce diplôme vous coûtera $\mathrm{C}$; au vu des salaires déclarés par les titulaires actuels de ce diplôme, vous gagnerez probablement S' sur les 20 ou 30 ans à venir ; S' - S mesure la plus-value salariale $\left(\mathrm{PV}_{\mathrm{s}}\right)$ apportée par le diplôme sur la période considérée; $\mathrm{PV}_{\mathrm{s}}-\mathrm{C}$ mesure le retour sur investissement (ROI), que l'on peut comparer entre différentes universités. Si l'on contraste ce que PayScale présente comme l'université la plus rentable avec celle qui apparait comme la moins rentable, on voit que la première, Harvey Mudd College (une école d'ingénieurs de Californie), rapporte un ROI sur 30 ans de 2113000 \$ pour des frais d'étude de $221700 \$$, alors que la dernière classée, Valley Forge College (une école religieuse de Pennsylvanie), coûtera 114100 \$ pour un retour sur investissement négatif de 178000 \$.

8 Pour ce qui nous concerne ici, la partie la plus instructive du rapport de PayScale vient de la page qui invite les étudiants potentiels à choisir non seulement entre différentes universités, mais entre différents parcours de spécialisation (majors). Même si la conception de l'enquête et du site met clairement au premier plan la question financière du ROI (Majors That Pay You Back), une page propose deux autres critères de sélection des parcours d'études : Popular Jobs by Majors, qui aide à aligner les études sur leurs débouchés professionnels, et Majors That Change the World, pour ceux aux yeux de qui « la signification du travail compte davantage que le montant du salaire».

9 Que nous donne à voir le classement des filières relativement aux revenus salariaux ? Un peloton de tête regroupe différents diplômes d'ingénieurs, de mathématiques appliquées et d'informatique, avec des salaires de milieu de carrière compris entre 160000 et 100000 \$ par an. La première science « humaine » est l'économie qui arrive au $15^{\mathrm{e}}$ rang avec $97000 \$$. Il faut ensuite descendre jusqu'aux $45^{\mathrm{e}}, 48^{\mathrm{e}}$ et $49^{\mathrm{e}}$ rangs pour trouver la philosophie, les sciences politiques et la linguistique avec environ $78000 \$$ (juste après la publicité). Littérature anglaise et histoire sont autour $\mathrm{du} 60^{\mathrm{e}}$ rang, avec environ 71000 \$, tandis que les études de français, d'allemand, l'anthropologie, la psychologie et les «Humanities» se regroupent autour du $80^{\circ}$ rang avec environ 
63000 \$. Histoire de l'art, beaux-arts, arts du spectacle, sociologie, études classiques et religieuses se situent autour $\mathrm{du} 100^{\mathrm{e}}$ rang avec environ $57000 \$$.

\begin{tabular}{|c|c|c|}
\hline Classement & Spécialisation (major) & Salaire mi-carrière \\
\hline 1 & Ingénierie pétrolière & $160000 \$$ \\
\hline 15 & Économie & $96700 \$$ \\
\hline 44 & Publicité & $79400 \$$ \\
\hline 45 & Philosophie & $78300 \$$ \\
\hline 48 & Sciences politiques & $77000 \$$ \\
\hline 49 & Linguistique & $76800 \$$ \\
\hline 59 & Littérature anglaise & $71400 \$$ \\
\hline 60 & Histoire & $71000 \$$ \\
\hline 76 & French Language & $66700 \$$ \\
\hline 82 & Humanities & $61800 \$$ \\
\hline 94 & Histoire de l'art & $59000 \$$ \\
\hline 95 & Sociologie & $58800 \$$ \\
\hline 96 & Théâtre & $58600 \$$ \\
\hline 100 & Beaux-arts & $58200 \$$ \\
\hline 104 & Classics & $57000 \$$ \\
\hline 115 & Interdisciplinary Studies & $53400 \$$ \\
\hline
\end{tabular}

$\mathrm{Si}$, à ne regarder que ce seul tableau de revenus salariaux, le résultat des courses ne paraît pas être disqualifiant pour les humanités, le concept technique de " retour sur investissement » éclaire ces données d'une lumière bien plus crue, qu'un long article de l'hebdomadaire The Economist résume assez brutalement. Après avoir relevé les beaux résultats des écoles d'ingénieurs, l'hebdomadaire britannique remarque que « les cours en arts et en humanités font voir un tableau plus mitigé » :

Tous [ces diplômes en arts] nourrissent sans doute l'âme, mais pas tous ne remplissent le portefeuille. Un diplôme en arts d'une université rigoureuse comme Columbia ou l'Université de Californie à San Diego est un très bon investissement. Mais un diplômé en arts de Murray State University dans le Kentucky peut s'attendre à gagner $147000 \$$ de moins sur 20 ans qu'un lycéen, une fois inclus ses frais de scolarité universitaire. Parmi les 153 diplômes en arts inclus dans l'enquête, 46 rapportent moins qu'un placement minimal sur 20 ans dans des bons du trésor. Et parmi ces 46,18 indiquent des retours négatifs sur investissement ${ }^{3}$. 
11 Si les arts et lettres ne remplissent pas forcément le compte en banque de leurs amants - on s'en doutait - peu importe finalement, dès lors que ces disciplines « nourrissent l'âme ». Les données rassemblées par PayScale montrent-elles dès lors une proportion inverse entre les taux de rémunération salariale et les taux d'épanouissement intellectuel, comme le laissent entendre à la fois l'article de The Economist et les excuses de Barack Obama attribuant à ses cours d'histoire de l'art certaines de ses joies existentielles ultérieures ? Pas exactement... Ce sont les infirmiers (premier rang) et les travailleurs sociaux (5) qui disent trouver le plus de sens dans leur carrière, suivis d'assez près par les ingénieurs des industries pétrolières (16, ceux-là mêmes qui sont les mieux payés), tandis que les premières sciences sociales n'apparaissent que vers la $40^{\mathrm{e}}$ place, les humanités et les arts étant tous réduits à la moitié inférieure du tableau (entre la $70^{\mathrm{e}}$ position et la $100^{\mathrm{e}}$ position), avec moins de la moitié des diplômés trouvant du sens dans ce qu'ils font.

\begin{tabular}{|c|c|c|}
\hline Classement & Spécialisation (major) & Satisfaction \\
\hline 1 & Infirmière & $85 \%$ \\
\hline 5 & Travail social & $78 \%$ \\
\hline 16 & Ingénierie pétrolière & $72 \%$ \\
\hline 43 & Sciences sociales & $60 \%$ \\
\hline 48 & Interdisciplinary Studies & $58 \%$ \\
\hline 58 & Sociologie & $55 \%$ \\
\hline 61 & Liberal Arts & $54 \%$ \\
\hline 66 & Linguistique & $53 \%$ \\
\hline 74 & Humanities & $50 \%$ \\
\hline 74 & French Language & $50 \%$ \\
\hline 79 & Littérature anglaise & $49 \%$ \\
\hline 79 & Histoire de l'art & $49 \%$ \\
\hline 86 & Histoire & $48 \%$ \\
\hline 86 & Beaux-arts & $48 \%$ \\
\hline 92 & Théâtre & $47 \%$ \\
\hline 109 & Philosophie & $44 \%$ \\
\hline 109 & Économie & $44 \%$ \\
\hline 124 & Classics & $36 \%$ \\
\hline
\end{tabular}


Ici aussi, il faut bien entendu prendre des précautions face aux questions posées par l'enquête et aux exclusions opérées. Les étudiants de licence qui auront trouvé le plus d'intérêt dans leur matière auront sans doute poursuivi leurs études au niveau gradué, et auront donc de ce fait été exclus de ces statistiques. La question posée est par ailleurs assez équivoque : ces chiffres donnent « le pourcentage de diplômés qui ont affirmé que leur emploi contribuait à améliorer le monde [the percentage of graduates with a specific major who reported that their job makes the world a better place]", ce qui est posé comme équivalent du « pourcentage de diplômés qui ressentent que leur emploi est porteur de sens [who feel their job is meaningful] ». Outre qu'on peut trouver du sens à une activité sans affirmer pour autant qu'elle rend le monde meilleur, on voit que cela mesure le taux de satisfaction envers l'emploi du diplômé, plus qu'envers le contenu de ses études. On comprend qu'un étudiant muni d'une licence en philosophie ou en lettres classiques ne trouve guère de sens dans son travail, si son emploi consiste à rédiger des rapports d'audits internes au sein d'un grand groupe financier - et on ne saurait en faire porter le blâme à son professeur philosophie ou de latin.

Quelles que soient les nombreuses tares qui vicient assez irrémédiablement la signification réelle des chiffres collectés par le rapport de Payscale, ces chiffres projettent une image qui n'est guère favorable aux disciplines relevant des humanités. Le site se fend certes d'une brochure de conseils, où Zachary First, détenteur de BA de philosophie (mais aussi d'un doctorat de Harvard) devenu directeur du Drucker Institute, souligne l'importance de développer une " passion pour le savoir et une réelle profondeur de connaissance sur quelque chose qui compte vraiment pour nous ${ }^{4} »$. Cette page dégoulinante de bons sentiments peine à compenser le message convergent asséné statistique après statistique : étudier les humanités, c'est dépenser beaucoup d'argent pour en gagner assez peu, voire pour en perdre beaucoup, et sans en tirer de satisfaction particulière. Les retours ne sont nulle part au rendez-vous des investissements.

\section{L'impasse de la professionnalisation}

Quelles leçons tirer pour la France de la petite phrase du président Obama et de la grande enquête de PayScale? Ne vivons-nous pas dans un monde complètement différent, où les études de licence ne coûtent pas $200000 €$, où (malgré quelques châtaigniers de la lie des hebdomadaires) la furie des classements n'a pas encore complètement colonisé des institutions d'éducation supérieure encore majoritairement publiques, où l'on regarde avec une condescendance amusée quiconque croirait sérieusement contribuer à make the world a better place? Contrairement aux USA, fer de lance du capitalisme sauvage, n'avons-nous pas mis en place des garde-fous pour protéger nos cultures humanistes à grands efforts d'exception culturelle, de parrainage étatique des Arts et de présidents littérateurs?

Le retour en France mérite d'être mis sous la lumière de deux citations collectées par Romuald Bodin et Sophie Orange dans leur ouvrage récent proclamant que L'université n'est pas en crise. Bien plus brutale et moins apologétique que Barack Obama, la ministre de l'Enseignement supérieur Geneviève Fioraso affirmait en mars 2013 que « dès la 
seconde, les jeunes doivent savoir que des filières comme l'histoire, la sociologie ou la psychologie connaissent des difficultés d'insertion». En France comme aux USA, les « projets d'avenir » des langues, littératures et sciences humaines doivent compter avec l'ombre de cet avertissement sur les « difficultés d'insertion ».

Bien que le risque de chômage diminue avec le niveau d'études, les écarts de taux de chômage selon les filières de formation sont élevés à tous les niveaux

Périmètre : population active diplômée au cours des dix dernières années

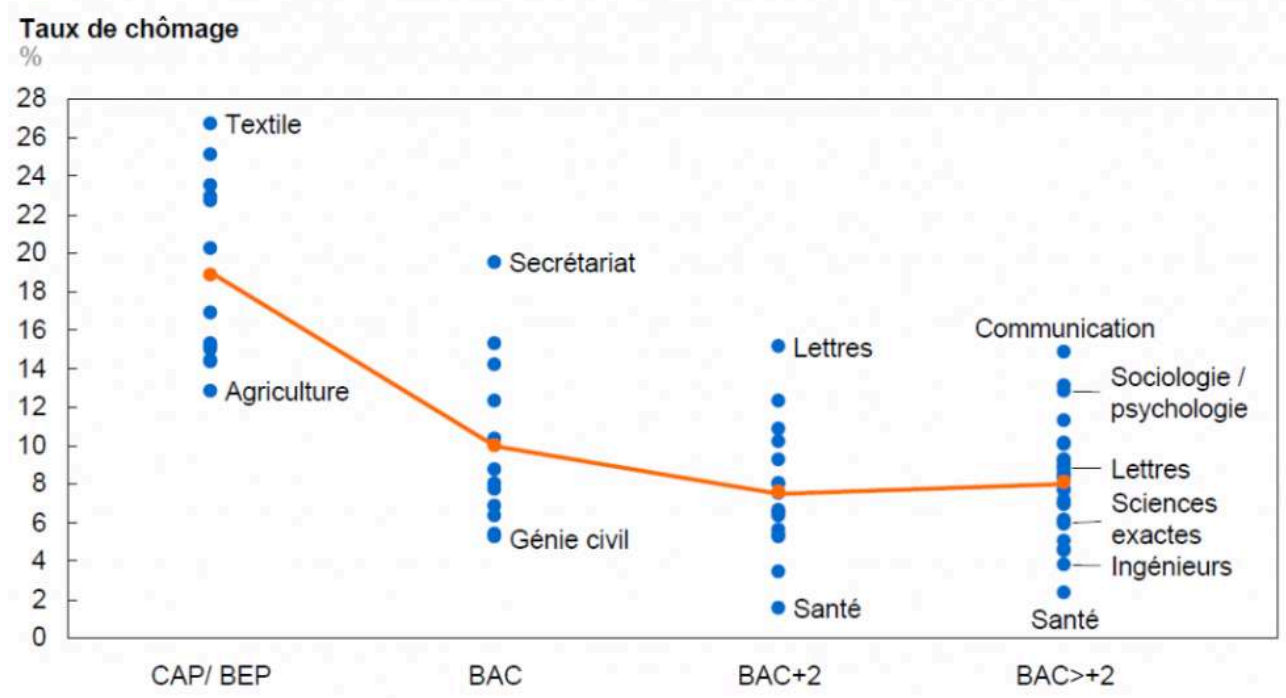

SOURCE : INSEE, synthèse des études sur l'emploi de 2003 à 2009 ; analyse du McKinsey Global Institute

Fig. 1. McKinsey Report, L'emploi en France 2020, mars 2012

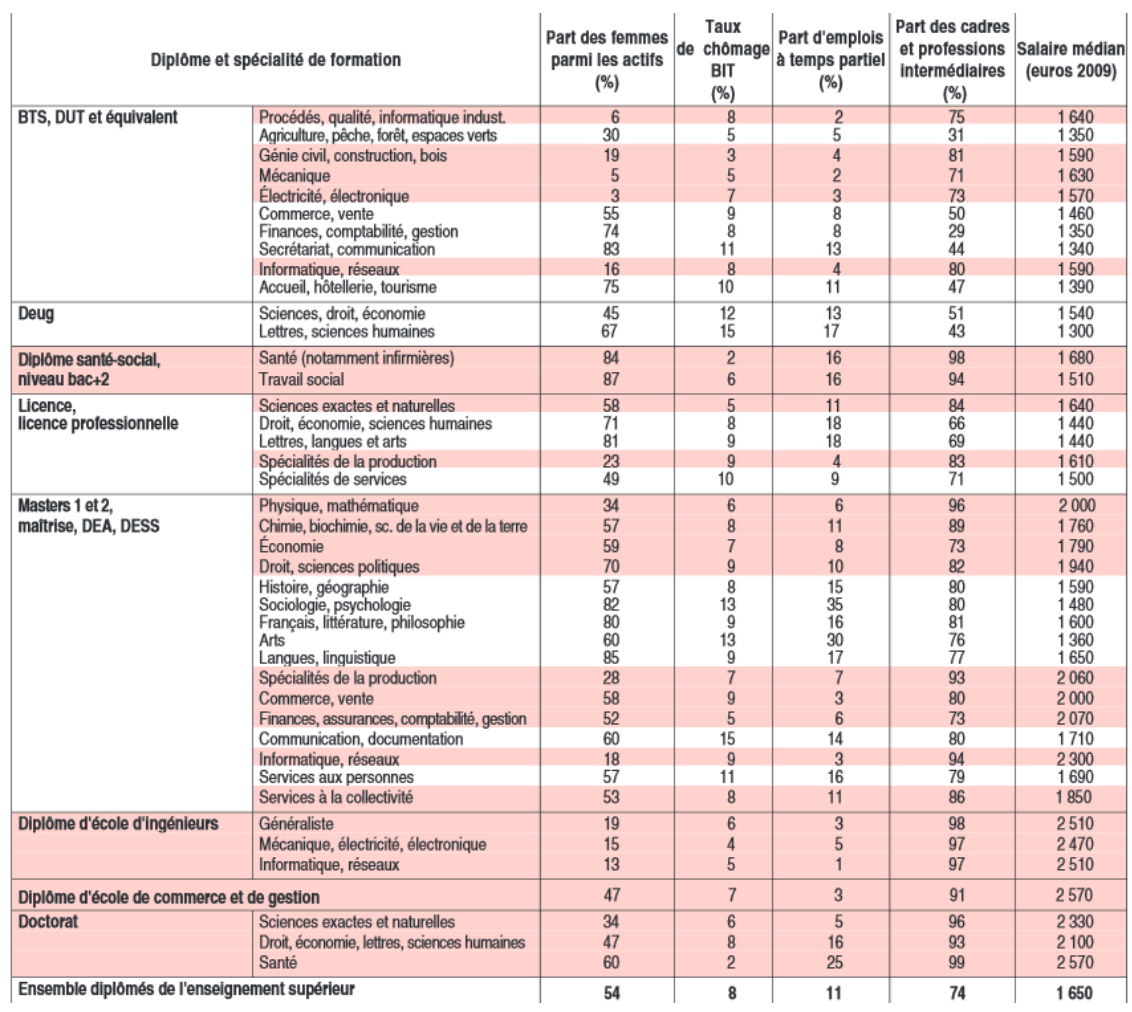


Fig. 2. Source : Insee Première, $\mathrm{n}^{\circ}$ 1313, octobre 2010 professionnalisation - partagé avec une remarquable continuité à travers les basculements de majorité gouvernementale. Ce qu'on désigne par le nom trompeur de « chômage » étant universellement érigé en ennemi public $n^{\circ} 1$ de la nation, n'est-il pas de toute évidence impératif et urgent de mieux ajuster nos formations aux besoins réels du marché de l'emploi? Les «Trente Glorieuses" pouvaient peut-être se permettre de multiplier les enseignements de philosophie et de littérature, sans se préoccuper des débouchés de tels diplômes, dès lors qu'une économie en expansion régulière absorbait tout et n'importe qui dans sa croissance euphorique. Mais «la crise » est passée par là, et le plus élémentaire réalisme nous impose de compter nos sous. De combien de critiques littéraires, de sociologues, d'anthropologues ou d'historiens de l'art peut donc avoir besoin la France? Quelques centaines, un petit millier? Tout ce que nos facultés de lettres, de sociologie ou d'histoire de l'art produisent au-delà de ces besoins réels relève du gaspillage, faisant de ces facultés autant d'« usines à chômeurs ».

Parce que la France préfère la bureaucratie verticale à l'ajustement réciproque des intérêts par les mécanismes de marché, ce sont des ministères qui, par évaluations, décrets et micro-management interposés, imposent de haut un strict alignement des parcours d'études sur les débouchés offerts par le marché actuel de l'emploi. Les universités en sentent directement le poids à travers les consignes et les critères d'évaluation imposés par les récentes vagues de l'AERES. Chaque master est sommé de se mettre au garde-à-vous pour indiquer clairement à quels types d'emplois conduira la formation qu'il met en place. S'il échoue à s'aligner sur un débouché précis et crédible, c'est qu'il travaille pour le compte de la grande usine à chômeurs - et que ses jours sont comptés.

Même si PayScale opère dans le cadre d'une auto-sélection par les agents intéressés, plutôt que par directives ministérielles, la logique est fondamentalement convergente entre les statistiques américaines du ROI Report et les injonctions de l'AERES. Des deux côtés de l'Atlantique, c'est la logique du retour sur investissement qui domine la structuration des parcours d'enseignement. Une formation ne mérite d'exister (d'être 
achetée par un étudiant / d'être financée par l'État) que si le marché actuel de l'emploi en rétribue les dépenses, que ce soit à travers une traduction directe en salaires individualisés (selon la logique de PayScale) ou que ce soit à travers l'optimisation planifiée des débouchés sur le marché de l'emploi (comme le demande l'AERES).

En quoi cet impératif de professionnalisation constitue-t-il une impasse pour l'enseignement supérieur, et plus généralement pour nos sociétés dans leur ensemble? La liste d'arguments est longue, mais j'en signalerai trop rapidement trois points principaux :

1. Tel qu'il est conçu actuellement, l'impératif de professionnalisation tend à enfermer les étudiants beaucoup trop tôt dans des parcours excessivement spécialisés, comme si les études supérieures n'étaient que des parcours d'apprentissages de métiers intellectuels, alors qu'il faut les concevoir comme des constructions de compétences très larges d'analyse, de réflexion, d'argumentation et d'expression.

2. L'impératif de professionnalisation aligne strictement l'éducation de nos populations sur les seules exigences du marché sous régime capitaliste néolibéral, alors même que la logique de ce marché est en train de nous conduire tous à l'abîme social (d'inégalités exacerbées) et écologique (d'autodestruction environnementale).

3. Parce que la professionnalisation - de même que son envers symétrique: le chômage n'est conçue qu'en termes de revenus salariaux, toute une énorme part de la productivité réelle de nos activités échappe complètement à l'étalon avec lequel on mesure la rentabilité des investissements éducatifs. La survie et le bien-être de nos sociétés dépend de plus en plus d'une énorme palette d'activités non-salariées (bénévolat associatif, care de proximité, auto-formation permanente, capacité de négociation, d'invention de solutions nouvelles à des problèmes inédits, «free labor » et «playbor » sur Internet). Parce que ces activités passent en dessous des radars de la professionnalisation et du marché du travail, elles restent administrativement et politiquement invisibles, et se voient menacées d'extinction $\mathrm{du}$ fait de leur invisibilisation parfaitement artificielle.

Pour prendre une analogie à la mode, l'obsession de la professionnalisation revient donc à ne mesurer l'utilité des abeilles qu'aux quantités et aux prix du miel qu'on peut tirer de leurs ruches pour le vendre sur le marché des édulcorants. Dès lors que la canne à sucre, la betterave ou l'aspartame peuvent se commercialiser plus avantageusement que le miel, on décidera que le maintien des ruches ne rapporte plus de retours suffisants sur investissement, et l'on en interrompra la production - cela de façon très « rationnelle " d'un point de vue étroitement comptable. L'effondrement actuel des populations d'abeilles nous fait toutefois comprendre que la principale production de richesse émanant des abeilles n'est pas à situer dans les profits facilement mesurables liés à la vente du miel, mais dans le travail diffus de pollinisation qui a longtemps échappé à toute forme de calcul, mais dont on s'aperçoit qu'il est proprement vital et inestimable.

Le décalage entre calculs de professionnalisation et contribution réelle à la prospérité sociale se pose bien entendu d'une façon particulièrement cruciale pour les disciplines relevant des humanités. Au-delà de quelques critiques littéraires, traducteurs, sociologues et anthropologues dûment professionnalisés, ce sont toutes nos populations qui doivent devenir (au moins un peu) critiques, traductrices, sociologues et anthropologues pour faire face aux problèmes de plus en plus complexes que posent nos modes de vie de plus en plus intriqués et interdépendants. Comme les chiffres de PayScale et les commentaires de The Economist, les exigences du ministère et de l'AERES étranglent à moyen terme les enseignements supérieurs proposés dans ces disciplines, 
parce que leur vocation est d'être bien davantage que simplement « professionnalisante ».

\section{L'excuse de l'épanouissement personnel} Il est indéniable que nos enseignements perdent en attractivité du fait de pressions extérieures qui poussent les étudiants à choisir des filières « rentables ». Mais il est plus que probable que nous sommes partiellement responsables de cette perte d'attractivité $\mathrm{du}$ fait de facteurs internes à nos disciplines, qui n'ont pas fait assez pour se rendre attirantes, pertinentes, importantes au sein des transformations rapides du monde 
actuel. Si nos effectifs baissent, c'est aussi parce que nous ne parvenons pas à donner une image assez exaltante de ce que nous faisons dans nos salles de classe. Au sein de l'énorme compétition attentionnelle qui régit notre accès à la culture, tout cours qui ne s'efforce pas de répondre directement à la question de son importance sociale s'expose de facto à passer à la trappe. On peut le regretter, mais il faut le constater - et en tirer les conséquences.

Un troisième registre d'action est moins velléitaire, mais peut-être plus important encore. Au lieu de nous complaire avec tant de nos collègues dans un discours qui dévalue systématiquement les capacités et motivations des jeunes générations, pourquoi ne pas faire une avance de confiance à ceux que Claude Burgelin appelait, il y a 25 ans déjà, ces "obstinés qui s'accrochent "? S'ils se retrouvent dans nos cours, envers et contre tout, c'est que quelque chose les y a poussés, dont nous devons aider à faire émerger la positivité et la force - qui résident toutefois bien davantage dans leurs désirs et leurs besoins que dans notre savoir. Un peu d'humilité de notre part, postulant une intelligence propre à ce qui nourrit le comportement de nos étudiants, serait peutêtre la meilleure façon de revaloriser nos filières. Si «nourrir l'âme » ne suffira pas à faire survivre institutionnellement les humanités, en revanche donner à l'âme de nos étudiants assez d'espace d'auto-détermination pour s'épanouir aussi puissamment et aussi librement que possible - voilà peut-être ce qui pourrait faire la spécificité et l'attrait de nos champs de recherche.

\section{L'illusion de l'individualisation}

La troisième tendance que partagent les situations françaises et américaines, illustrées respectivement par nos politiques ministérielles et par les statistiques de PayScale, est plus profonde que les deux autres, et se situe bien à la racine des problèmes discutés jusqu'à présent. Sous l'inertie d'une pression multiséculaire qui remonte au moins à l'émergence de l'économisme capitaliste à l'époque des Lumières, tous nos systèmes sociaux se sont progressivement réorganisés autour de pertinences de plus en plus strictement individualisées. Le rapport salarial en est bien entendu un exemple emblématique, particulièrement sous sa modalité néolibérale, mais on voit depuis quelques années nos systèmes de retraite passer également de la couverture collective par répartition à des assurances et fonds de pensions individualisés. Comme l'ont montré Michel Foucault et Pierre Bourdieu, parmi bien d'autres, l'école a été un facteur essentiel de ce mouvement d'individualisation des performances et des compétences, dans la mesure où elle assigne à chacun une palette de notes et de rangs qui le positionnent très précisément au sein des diverses compétitions à travers lesquelles se joue «la réussite sociale ». Chacun(e) de nous, dès lors que nous acceptons de jouer le jeu des notes, fonctionne comme un agent direct de cette énorme individualisation multiséculaire de nos rapports sociaux - avec les grandes écoles y jouant sans doute un rôle encore plus caricatural à travers leur habitude de classer sur une seule échelle de réussite leurs diplômés de l'année.

Or cette individualisation nous conduit aujourd'hui clairement dans le mur social et écologique. L'échelle des intérêts individualisés n'est plus du tout en phase avec des phénomènes comme les centrales nucléaires, les délocalisations de production ou le dérèglement climatique : et du point de vue des distances spatiales et de celui des horizons temporels, l'individualisation exacerbée de nos modes de décision conduit au 
suicide collectif. Or, au lieu d'aller à l'encontre des tendances perverses de cette individualisation forcenée, la plupart des « réformes de l'éducation " concoctées ces dernières années en accélèrent la logique calamiteuse.

PayScale en fournit bien entendu une illustration caricaturale, mais c'est seulement le sommet d'un iceberg présent sous beaucoup d'autres phénomènes. Si les étudiants américains sont conduits à calculer le retour sur investissements de leurs quatre ans d'université, c'est parce que le coût de leur éducation a été strictement individualisé sous forme de frais d'inscription et de dette étudiante. Encore une fois, il ne s'agit pas que d'une bizarrerie américaine : l'Angleterre vient de s'engouffrer dans un processus similaire en à peine quelques années, et l'on entend régulièrement en France des appels à aller dans ce sens, avec des premières percées comme la hausse des frais d'inscription de Sciences Po Paris.

Contre cette tendance lourde et autodestructive, l'urgence est d'affirmer et de faire reconnaître la nature de bien commun des acquis éducatifs ${ }^{6}$. On entend souvent dire qu'il faut un village pour éduquer un enfant; il faut ajouter que c'est tout le village qui s'enrichit des compétences de chacun de ses membres. Et il faut surtout en tirer les conséquences pratiques: l'éducation doit être conçue, traitée, cultivée, soignée et financée comme un bien commun, au même titre que l'air que nous respirons, l'eau que nous buvons et la pollinisation accomplie par les abeilles. Non sans ironie, on trouve sur le site de PayScale l'une des affirmations les plus saisissantes de la valeur commune de l'éducation, attribuée à Victor Hugo, côtoyant ici le président Obama et de Tom Hanks, affirmation selon laquelle " ouvrir une école, c'est fermer une prison ». On sait que les taux d'incarcération sont encore plus sensibles à l'obtention de diplômes que les taux de chômage. Parmi le travail de pollinisation invisible accompli par les études supérieures, il faudrait aussi compter le fait de moins recourir aux crimes pour promouvoir ses intérêts individuels. Or comme l'air que l'on respire, cette «noncriminalité » ne se voit pas et ne se mesure que très difficilement. Combien d'autres compétences ou modes de comportements ne sont-ils pas également inculqués de façon diffuse par des cursus de langues, de lettres, de philosophie, de psychologie ou de sociologie?

La véritable question est donc de savoir comment valoriser cette productivité diffuse de l'éducation en général, et de certaines filières apparemment peu rentables en particulier. Car les retours (communs) sur investissement (publics) sont bien là - sauf qu'ils échappent aux appareils de mesure auxquels les réduit une approche étroitement économiciste. Le problème n'est donc pas que les étudiants «fassent les mauvais choix » en s'orientant vers certaines filières plutôt que d'autres. Le problème vient de ce que des appareils d'orientation - illustrés en l'occurrence par PayScale et par les directives ministérielles - individualisent artificiellement et étroitement leurs motivations en termes de soumission aux logiques actuelles du marché de la dette, du placement financier ou de l'emploi, tel que ce marché est régi lui-même par les calculs de profit capitaliste.

\section{L'ubiquité de la médiation}

Pour peu qu'on les redéfinisse comme des pratiques réfléchies de l'interprétation, les humanités sont amenées à occuper une place centrale au sein de nos transformations sociales actuelles. Que faisons-nous dans nos cours de langues, de lettres, d'histoire de 
l'art, de sociologie, de psychologie, d'histoire, d'anthropologie ou de philosophie, sinon essayer de comprendre comment les sociétés humaines produisent le sens qui leur permet de s'orienter dans le monde? Il n'y a certes pas de profession spécifique, inscrite au registre du commerce, qui serve de débouché précis pour ce type d'apprentissage - d'où l'invisibilisation de notre productivité diffuse au sein des appareils de mesure et d'orientation actuels. Et pourtant, ce que nous faisons avec nos étudiants touche à la base même de toutes nos autres activités productives - à leur infrastructure sémantique et affective de valorisation. Si tout être humain décode et interprète en permanence les données sensorielles qui lui parviennent de son environnement, notre spécificité est de constituer des espaces de réflexion critique sur ces pratiques interprétatives omniprésentes. Ces pratiques réfléchies de l'interprétation nous font à la fois toucher du doigt, valoriser, adapter et cultiver activement la constitution même de notre commun.

Le repositionnement des études littéraires au sein d'«études de media comparés ", catégorie proposés par Kathernie Hayles, professeur de littérature à l'université de Duke aux États-Unis ${ }^{7}$, fournit un cadre renouvelé, inclusif et fédérateur, nous permettant de continuer à faire ce que nous faisons de bien, ainsi que de concevoir de nouvelles activités, plus directement en phase avec les évolutions sociales en cours - dans une perspective d'échanges mutuellement enrichissants avec certaines disciplines parentes et voisines.

Les études de media comparés reposent sur trois principes étroitement liés entre eux :

- Étudier nos objets d'investigation en tant qu'ils constituent des media: qu'on travaille sur des poèmes latins, sur des tragédies raciniennes, sur des romans postcoloniaux, mais aussi sur des films, des séries télévisées, des scénographies ou des chorégraphies, tous nos objets d'études et d'enseignements peuvent être approchés en tant que media ${ }^{8}$, pour autant qu'on infléchisse la définition courante de ce terme en lui ajoutant la dimension à la fois inclusive, précise et stimulante récemment proposée par Jussi Parikka :

Les media consistent en une action de plier le temps, l'espace et les agentivités ; ils ne sont pas la substance ou la forme à travers lesquelles des actions prennent place, mais un environnement de relations dans lequel le temps, l'espace et les capacités d'action émergent'.

- Étudier la spécificité de chacun de nos objets d'investigation en tant qu'ils constituent un medium singulier. Si le premier mouvement consiste à redécrire nos objets d'étude en faisant apparaitre ce qui les qualifie comme media (en général), le deuxième mouvement consiste à identifier et à faire reconnaître ce qui distingue les propriétés médiologiques d'un poème latin de celles d'un roman postcolonial, d'une série télévisée, d'une chorégraphie, ou d'un autre poème latin. Le comparativisme peut porter bien entendu sur des comparaisons entre différents media, différentes cultures, différentes langues, différents genres, différentes écoles, différents courants, différentes périodes, mais à l'intérieur de chacune de ces classes, il vise aussi à faire apparaitre la singularité médiologique de chacune des œuvres analysées. Ce comparativisme invite également à contraster (pour leur enrichissement mutuel) différentes approches méthodologiques (narratologie, études subalternes, thématique, génétique textuelle, éco-critique, etc.) essayées sur la même œuvre singulière. Ce que nous comparons à travers nos diverses études, c'est bien - pour reprendre les termes de Parikka - différentes façons que proposent les œuvres de «plier le temps, l'espace et les agentivités ".

- Concevoir nos études comparatives sous l'horizon d'une archéologie des media inscrivant la prétendue nouveauté des media numériques dans la perspective à long terme de pratiques 
multiséculaires de médiation. L'affirmation d'une pertinence sociale actuelle des études de media comparés peut s'appuyer sur un besoin urgent de mieux identifier et de mieux comprendre - mais aussi de relativiser - ce que nos " nouveaux media » (le numérique, Internet, la gouvernementalité algorithmique) contiennent de promesses et de menaces dans l'évolution constante de notre anthropogenèse. Que nous pratiquions les tweets, ayons notre page Facebook, ou que nous nous en méfiions comme de la peste, nous occupons une place stratégiquement essentielle pour aider notre société à mieux comprendre et maitriser les dispositifs techniques et symboliques qui régissent nos interactions. Les études littéraires élaborent depuis vingt-cinq siècles des outils d'analyse qui restent de très loin les plus fins à ce jour pour rendre compte de l'efficacité et de la subtilité des médiations symboliques qui contribuent à plier le temps, l'espace et les agentivités humaines. La rhétorique antique, l'exégèse médiévale, la philologie issue de la Renaissance, la critique historique pratiquée à partir du XVIII ${ }^{e}$ siècle, la didactique émanée des réformateurs pédagogiques de la modernité naissante, ainsi que tous les courants disciplinaires qui se sont succédé depuis, ont accumulé un arsenal méthodologique et conceptuel qui nous met en position idéale pour aider nos contemporains à resituer nos prétendues nouveautés dans des évolutions à très long terme, ainsi qu'à mieux identifier et à mieux mesurer les implications des quelques innovations véritablement inédites dont nous sommes témoins et acteurs. C'est à ce mouvement de vaet-vient entre les innovations numériques et le recul historique apporté par la connaissance des médiations symboliques du passé lointain qu'est dédiée la discipline émergente de l'archéologie des media - dont il n'est guère étonnant de constater que les pionniers émanent presque tous de formations littéraires (Marshall McLuhan, Friedrich Kittler, Siegfried Zielinski, Daniel Bougnoux, Stefan Andriopoulos).

37 Ainsi redéfinies, nos études peuvent revendiquer un positionnement épistémologique et «professionnalisant » crucial au sein de nos pratiques sociales. Bien au-delà du supplément d'âme et d'une meilleure compréhension de l'impact des nouveaux media, nous nous positionnons comme des spécialistes de la médiation, à la fois sur le plan de son étude analytique et sur celui de ses modulations pratiques. En des termes vulgarisés par Bruno Latour, notre travail consiste à faire sentir et comprendre en quoi tout « intermédiaire " (s'efforçant d'être aussi efficace et transparent que possible) contient la présence d'un "médiateur » (doté de son agentivité propre). Une panne ou une crise symbolique ne sont rien d'autre que l'irruption du médiateur outrepassant et brouillant son rôle attendu de simple intermédiaire ${ }^{10}$.

Un tel repositionnement nous permet de développer des argumentaires servant de contre-feux au laminage de nos effectifs sous l'impératif de professionnalisation: en analysant et en enseignant la complexité et les subtilités des pratiques de médiations, nous opérons à une échelle vouée à rester invisible du point de vue des débouchés professionnels. La médiation n'est localisable nulle part comme telle dans la liste des métiers ; et pourtant, la médiation est partout, mais sous forme diffuse, puisque nul ne peut être un bon collaborateur de quelque entreprise que ce soit s'il n'a pas acquis une certaine compétence de médiateur. Notre fonction sociale est d'apprendre à mieux parler les langues de la médiation - à l'heure où nos chaînes d'actions sont de plus en plus en plus longues, complexes, intriquées, et donc de plus en plus fragilisées par les difficultés de médiation. Nos parcours ne sont ni plus ni moins "professionnalisants » que ceux qui enseignent à (mieux) parler ou écrire - dont il serait absurde de mesurer «les débouchés" au seul nombre des discoureurs professionnels. Qu'elles portent sur les arts du spectacle, l'histoire littéraire, la didactique, la traduction ou la 
création poétique, les études de media comparés proposent un apprentissage de la médiation comme médiation - et nul savoir n'est appelé à être aussi précieux dans les transformations actuelles de nos sociétés.

Loin de relever de la seule "joie», ou du seul épanouissement individuel, les humanités, conçues comme pratiques réfléchies de l'interprétation et comme études des médiations en tant que médiations, touchent au cœur de ce qui nous fait tenir ensemble. Elles n'ont littéralement pas de prix - non au sens où elles échapperaient à toute mesure, mais au sens où les prix du marché sont incapables d'en rendre compte. Leur prix apparaît clairement quand le sens du commun se délite, dans les cas de conflits religieux et de guerres civiles. C'est à nous qu'il appartient de faire sentir ce prix avant de pâtir de leur délitement - non seulement pour défendre nos intérêts corporatistes, mais surtout pour assurer notre survie commune.

\section{NOTES}

1. Remarque du 30 janvier: « Folks can make a lot more potentially with skilled manufacturing or the trades than they might with an art history degree. Now, nothing wrong with an art history degree - I love art history. So I don't want to get a bunch of emails from everybody. I'm just saying, you can make a really good living and have a great career without getting a four-year college education, as long as you get the skills and training that you need. » Réponse de février : "Let me apologize for my off-the-cuff remarks. I was making a point about the jobs market, not the value of art history. As it so happens, art history was one of my favorite subjects in high school, and it has helped me take in a great deal of joy in my life that I might otherwise have missed» (citations et vidéo disponibles, entre autres, sur le site <http:// www.politico.com/story/2014/02/president-obama-apologizes-to-art-history-

professor-103626.html>, consulté le $1^{\mathrm{er}}$ mai 2014).

2. [En ligne] : <http://www.payscale.com/college-roi/>. Toutes les données ci-dessous sont tirées de ce site, consulté le $1^{\mathrm{er}}$ mai 2014.

3. « Is college worth it?», The Economist, 5 avril 2014, p. 40.

4. Z. First, "When the Humanities Are Worth It " dans le dossier Examining the Value of a College Degree, p. 12. [En ligne] : <http://www.payscale.com/content/value-college-degree.pdf> (consulté le $1^{\mathrm{er}}$ mai 2016)

5. R. Bodin et S. Orange, L'université n'est pas en crise, Bellecombe-en-Bauges, éd. du Croquant, 2013, p. 57. La citation de Geneviève Fioraso vient de Le figaro.fr en date du 20 mars 2013 ; celle de Claude Burgelin vient de Le Monde, 6 novembre 1979.

6. Voir sur ces questions, P. Dardot et C. Laval, Commun. Une révolution du XXI e siècle, Paris, La Découverte, 2014.

7. Ce syntagme ainsi que le cadrage proposé ici s'inspirent du travail de Katherine Hayles qui promeut des Comparative Media Studies dans son ouvrage de 2012, How We Think. Digital Media and Contemporary Technogenesis, University of Chicago Press, 2012. Voir également la traduction de cet ouvrage : Lire et penser en milieux numériques : attention, récits, technogenèse, trad. de l'anglais par C. Degoutin, Grenoble, Ellug, 2016.

8. À la suite de Thierry Bardini, on distinguera la graphie « media » (sans accent ni -s), désignant les objets techniques et symboliques considérés dans leurs propriétés médiologiques, tels que l'humanité en a produits depuis des millénaires, de la graphie « médias », désignant de façon plus 
restreinte les mass-media (presse, radio, télévision), tels qu'ils se sont développés depuis le XIX ${ }^{\mathrm{e}}$ siècle. Voir Th. Bardini, «Entre archéologie et écologie: Une perspective sur la théorie médiatique ", dans Multitudes, $\mathrm{n}^{\circ} 62$, 2016/1. [En ligne]:<https://www.cairn.info/revuemultitudes-2016-1-p-159.htm>

9. J. Parikka, « Media Ecologies and Imaginary Media: Transversal Expansions, Contractions, and Foldings ", The Fibreculture Journal, $\mathrm{n}^{\circ}$ 17, 2011, p. 35. [En ligne] : <http://fibreculturejournal.org> 10. Voir sur ce point, B. Latour, «Les médias sont-ils un mode d'existence? ", INA Global, $\mathrm{n}^{\circ} 2$, juin 2014, p. 146-152. [En ligne]:<http://www.yvescitton.net/wp-content/uploads/2014/09/ LATOUR-MediasModesExistence-Juin2014-Txt.pdf>

\section{RÉSUMÉS}

Qu'est-ce donc qui fait la valeur de la littérature et de son enseignement ? On peut tenter de répondre à cette question depuis l'intérieur, à partir des expériences de lecteur, d'enseignant ou de théoricien. On peut aussi tenter de comprendre par quels mécanismes et par quels discours économico-politiques les études littéraires se trouvent « valuées » depuis l'extérieur. Cet article observe la façon dont certains modes de classement des universités nord-américaines en termes d'investissement financier et certains discours européens sur le besoin de "professionnaliser » les cursus universitaires partagent quelques postulats similaires qui, au nom d'une certaine évaluation des pratiques littéraires, risquent de les évider de leur valeur réelle.

What is the value of (teaching) literature? This question can be addressed from the inside of our discipline, with arguments based on our experience as reader, teacher or theorist. It can also be approached from the perspective of the institutional devices and the economico-political discourses that structure the actual processes of valuation which determine the (market) value of literary studies. This paper analyzes how certain financial tools provided to measure the return on investment expectable from literary studies in North America converge with European discourses on "professionalization" in higher education, as both threaten to hollow out the real value of literary studies in the name of measuring their economic value.

\section{AUTEUR}

\section{YVES CITTON}

Yves Citton est professeur de littérature et media à l'université Paris 8, après avoir enseigné à l'université Grenoble Alpes et à l'université de Pittsburgh (USA). Il co-dirige la revue Multitudes et a publié récemment Contre-courants politiques (Fayard, 2018), Médiarchie (2017) et Pour une écologie de l'attention (2014) aux éditions du Seuil, ainsi que Zazirocratie (2011) et Mythocratie (2010) aux éditions Amsterdam. Ses articles sont en accès libre sur <www.yvescitton.net>. 